\title{
Post-stroke infection in acute ischemic stroke patients treated with mechanical thrombectomy does not affect long-term outcome
}

\author{
Klaudia Nowak ${ }^{1}$, Justyna Derbisz ${ }^{1}$, Jan Pęksa², Bartłomiej Łasocha3 ${ }^{3}$ Paweł Brzegowy ${ }^{3}$, Joanna Slowik ${ }^{4}$, \\ Paweł Wrona ${ }^{1}$, Roman Pulyk ${ }^{1}$, Tadeusz Popiela ${ }^{3}$, Agnieszka Slowik ${ }^{1}$
}

${ }^{1}$ Department of Neurology, Jagiellonian University Medical College, Krakow, Poland

2Department of Cardiology, Interventional Cardiology and Hypertension, University Hospital, Krakow, Poland

${ }^{3}$ Department of Radiology, University Hospital, Krakow, Poland

${ }^{4}$ Department of Dental Prophylaxis and Experimental Dentistry, Jagiellonian University Medical College, Krakow, Poland

Adv Interv Cardiol 2020; 16, 4 (62): 452-459

DOI: https://doi.org/10.5114/aic.2020.101771

\begin{abstract}
Introduction: The impact of an infection that requires antibiotic treatment (IRAT) after an acute ischemic stroke (AIS) treated with mechanical thrombectomy (MT) remains unclear.

Aim: Here, we studied the prevalence and the profile of IRAT in patients with AIS treated with MT, aiming to identify predictive factors and prognostic implications at 90 days after stroke.

Material and methods: We analyzed parameters available within $24 \mathrm{~h}$ after AIS including demographics, risk factors, National Institutes of Health Stroke Scale (NIHSS) upon admission and $24 \mathrm{~h}$ later, hemorrhagic transformation (HT) on computed tomography, and several clinical and biochemical markers. The outcome measures were the modified Rankin Scale (mRS) 0-2 and 90 days post-stroke mortality.

Results: We included 291 patients; in 184 (63.2\%) patients MT was preceded by intravenous thrombolysis (IVT), and 83 (28.5\%) patients developed IRAT. Multivariate analysis showed that male sex and hemorrhagic transformation on CT taken $24 \mathrm{~h}$ after stroke increased the risk of IRAT. We found that younger age, male sex, lower delta NIHSS, shorter time from stroke onset to groin puncture, better recanalization and a lack of hemorrhagic transformation on CT taken $24 \mathrm{~h}$ after stroke favorably affected outcome at day 90 . Multivariate analysis showed that older age, higher delta NIHSS, unknown stroke etiology and lack of treatment with IVT were independent predictors of death up to day 90. Infection that required antibiotic treatment did not enter in the models for the studied outcome measures.

Conclusions: In AIS patients treated with MT, IRAT is not an independent factor that affects favorable outcome or mortality 90 days after stroke.
\end{abstract}

Key words: mortality, acute ischemic stroke, infections, antibiotic therapy, mechanical thrombectomy, long-term outcome.

Su m m a ry

To the best of our knowledge, no study has yet evaluated the prevalence and the profile of infection that requires antibiotic treatment (IRAT) or parameters affecting the risk of IRAT and its prognostic significance in patients with acute ischemic stroke (AIS) treated with mechanical thrombectomy (MT). We included 291 AIS patients and 83 (28.5\%) developed IRAT. We found that IRAT was not an independent factor affecting favorable outcome or mortality 90 days after stroke; however, we confirmed the prognostic significance of commonly accepted parameters such as age, sex, neurological deficit assessed by NIHSS score, time from stroke onset to groin puncture, recanalization rate as measured by the Thrombolysis in Cerebral Infarction ( $\mathrm{TICl}$ ) score, and hemorrhagic transformation of ischemic lesion on $\mathrm{CT}$ after the procedure.

\section{Introduction}

Ischemic stroke is an important cause of morbidity and mortality worldwide with a considerable social and economic burden [1]. Since 2015, mechanical thrombectomy (MT) has been an approved treatment option for acute ischemic stroke (AIS) patients with their acute clot located in a large brain artery and who do not respond to

\section{Corresponding author:}

Klaudia Nowak MD, Department of Neurology, Jagiellonian University Medical College, 2 Jakubowskiego St, 30-688 Krakow, Poland,

e-mail: claudianowak164@gmail.com

Received: 23.09.2020, accepted: 4.11.2020. 
intravenous thrombolysis (IVT) or who are outside their therapeutic window for IVT or for whom IVT is contraindicated [2]. MT markedly improves functional outcome of AIS compared to other treatment options [3]. Time from stroke onset to groin puncture is an important modifiable parameter affecting prognosis of patients treated with MT [4]. The question arises whether the efficacy of MT can be improved even more by identifying other modifiable factors that affect outcome.

Infection that requires antibiotic treatment (IRAT) is a common medical complication in AIS patients [5]. Several parameters that increase the risk of infection in AIS patients have been identified including age, sex, several comorbidities, cognitive alterations, preadmission dependency, speech problems, dysphagia, stroke severity, etc. [6-11]. Infection in AIS correlates significantly with longer in-hospital stay, higher medical costs [12, 13], and poor prognosis and mortality $[14,15]$.

To the best of our knowledge, no study has yet evaluated the prevalence and the profile of IRAT or parameters affecting the risk of IRAT and its prognostic significance in patients treated with MT. Knowledge about this important clinical aspect of AIS would be helpful in establishing standards of prevention.

\section{Aim}

We evaluated the prevalence and the profile of IRAT in AIS patients treated with MT. We also studied whether IRAT affected long-term favorable outcome defined by the modified Rankin Scale (mRS) score from 0 to 2 or mortality at day 90.

\section{Material and methods}

We retrospectively analyzed data prospectively collected from 291 patients with AIS who underwent MT at the Comprehensive Stroke Center in Krakow in the period from January 2013 to August 2019. The study was approved by the Jagiellonian University Ethical Committee (KBET 54/B/2007). We received written informed consent from all participants. All procedures were performed in accordance with the Declaration of Helsinki [16]. Stroke diagnosis was consistent with the WHO definition [17]. Details of patients' check-ups have been described previously [4].

In the present study we examined the following parameters: (1) demographics including age and sex; (2) stroke risk factors including hypertension, diabetes mellitus, ischemic heart disease, atrial fibrillation (AF), smoking status (smokers were those who were smoking while they were recruited into the study or those who had smoked in the past); (3) clinical data such as the $\mathrm{TICl}$ scale after the procedure [18], time-lapse from stroke onset to groin puncture (SO-GP), and stroke severity on admission and $24 \mathrm{~h}$ later according to the $\mathrm{Na}$ tional Institutes of Health Stroke Scale (NIHSS) [19]. Del- ta NIHSS was defined as the difference between NIHSS score tested $24 \mathrm{~h}$ after MT and upon hospital admission; low scores reflect improvement, and high scores indicate deterioration. We also measured post-treatment hemorrhagic transformation on computed tomography (CT) taken $24 \mathrm{~h}$ after stroke (patients were categorized as with or without bleeding), body temperature on admission, and stroke etiology according to the Trial of Org10172 in Acute Stroke Treatment (TOAST) criteria [20]. For this project the participants were classified as those with unknown etiology and those with a defined etiology (large vessel disease stroke, small vessel disease stroke, cardio-embolic stroke or stroke due to rare etiology).

We studied the occurrence of infection that required antibiotic treatment during hospitalization (IRAT). Diagnosis of infection was based on clinical symptoms, e.g., fever, laboratory tests, microbiological assays, and chest $X$-rays. We considered the following types of infection: pneumonia, urinary tract infection, thrombophlebitis, and wound infection. We distinguished two types of pneumonia: community acquired pneumonia (CAP) and nosocomial pneumonia. Routinely in our hospital, first line treatment for patients with CAP is ceftriaxone and for patients with nosocomial pneumonia is ceftriaxone and levofloxacin. If these antibiotics are not effective we modify the treatment according to the antibiogram. For patients with urinary tract infection the first-line treatment is cefuroxime. If this antibiotic is not effective we modify the treatment according to the antibiogram. Fasting blood samples on the first morning after admission were also tested for white blood cell count (WBC), hemoglobin levels, glucose levels, creatinine, TSH, low-density lipoprotein (LDL), high-density lipoprotein (HDL), and triglycerides. We also studied treatment with IVT prior to MT and the following treatments before AIS: antiplatelets, anticoagulants, and statins.

The outcome measures were the modified Rankin Scale [21] (mRS) 0-2 and mortality 90 days after stroke.

\section{Statistical analysis}

Clinical characteristics and outcomes between the groups were compared using unpaired Student's $t$-test or the $\chi^{2}$ test where appropriate. To identify potential independent predictors of outcome, variables with $p$-values $<0.05$ in the univariate regression analyses for IRAT, favorable outcome or death were subsequently included in multivariate regression analyses.

All statistical calculations were performed by Statistica software version 12.5 (TIBCO software INC). P-values below 0.05 were considered statistically significant.

\section{Results}

MT was performed in 291 patients. Infection that required antibiotics during hospitalization was seen in 
83 (28.5\%) patients: 51 (17.5\%) patients developed pneumonia, 28 (9.6\%) developed urinary tract infections, $1(0.3 \%)$ patient developed wound infection, 1 (0.3\%) patient developed sepsis, and 2 (0.7\%) patients developed an infection of unclear origin. Eleven (21.6\%) patients developed CAP, and 40 (78.4\%) developed nosocomial pulmonary infection. Ten (19.6\%) patients required an antibiogram, since they did not respond to first-line antibiotics. The cultures revealed the following pathogens: Staphylococcus aureus MSSA (3); Staphylococcus aureus MRSA (2); Klebsiella pneumoniae ESBL (+) (2); Klebsiella pneumoniae (1); Escherichia coli (1); Enterobacter cloacae (1). In each case antibiotics were used according to the antibiogram. Nineteen out of 28 patients with urinary tract infection $(67,9 \%)$ responded to the first-line antibiotic. In the remaining 9 patients the cultures revealed the following pathogens: Escherichia coli (5); Escherichia coli ESBL (+) (1); Proteus mirabilis (1); Enterococcus faecalis (1); Staphylococcus aureus MSSA (1). In each case antibiotics were used according to the antibiogram. The blood culture of the patient with sepsis revealed Staphylococcus aureus and the patient was treated according to the antibiogram. Cultures from urine, blood and from bronchoalveolar lavage from the 2 patients with infection of unclear origin did not show any pathogens. Patients were treated with ceftriaxone and levofloxacin.

Patients who developed IRAT were older and more often were men. Univariate analysis showed that several features correlated with IRAT: diabetes mellitus, ischemic heart disease, AF, higher delta NIHSS, hemorrhagic transformation on CT taken $24 \mathrm{~h}$ after stroke, antiplatelet treatment, statin use before stroke, and higher glucose levels on the next day after admission (Table I). Multivariate analysis showed that male sex and hemorrhagic transformation on CT taken $24 \mathrm{~h}$ after stroke increase the risk of IRAT (Table II).

Here, 154 (52.9\%) patients had favorable outcomes at day 90 . Univariate analysis showed that patients with a favorable outcome were significantly younger, more often male, and with lower rates of diabetes mellitus. They had a significantly better recanalization rate, shorter time from stroke onset to groin puncture, and lower delta NIHSS than those with a poor outcome. Patients with favorable outcomes were less likely to have a hemorrhagic transformation on $\mathrm{CT}$, more likely to be treated with IVT and less likely to develop IRAT. They also had higher hemoglobin levels and lower glucose levels the next morning (Table I).

Multivariate analysis showed that younger age, male sex, lower delta NIHSS, better recanalization as graded by the $\mathrm{TICl}$ score, shorter time from stroke onset to groin puncture, and lack of hemorrhagic transformation on CT taken $24 \mathrm{~h}$ after stroke favorably affected outcomes at day 90. Infection that required antibiotic treatment did not affect this outcome (Table II).
There were 63 (21.6\%) deaths within 90 days. Patients who died more often were diagnosed with stroke of unknown origin while those who survived more often had a diagnosis of large vessel disease stroke (Table I). Univariate analysis showed that patients who died were older. Compared to their surviving counterparts, patients who died had worse recanalization as graded by $\mathrm{TICl}$ score, higher delta NIHSS, more frequently presented with hemorrhagic transformation on CT taken within $24 \mathrm{~h}$ after treatment and more often had stroke of unknown etiology. They less frequently had intravenous thrombolysis and they more often used statins before stroke and were more likely to develop IRAT. They had lower hemoglobin levels, higher WBC counts, and higher glucose levels the next morning after admission. Multivariate analysis showed that older age, higher delta NIHSS, unknown stroke etiology and lack of treatment with IVT were independent predictors of death up to day 90 .

\section{Discussion}

Many studies in patients with AIS, irrespectively of their acute treatment method [14, 15, 22-24], focus on analyzing the prevalence, the profile, and the prognostic significance of infection. Infection in AIS is an important aspect of stroke clinics, i.e., with respect to complications, lengths of hospital stay, or prognosis; however, clinical trials have shown that preventive antibiotic therapy did not improve functional outcome in relatively unselected AIS patients $[25,26]$.

To the best of our knowledge, the clinical characteristics of infection in AIS patients treated with MT have not yet been studied. MT is clearly better than other AIS therapies with a number needed to treat (NNT) less than three for improved functional outcome [3]; thus, the prognosis in AIS treated with MT may be determined by a different set of factors relative to other subgroups of AIS patients. For comparison, the NNT in AIS patients treated with IVT within 90 min after stroke onset is 5; this value is nine for those treated 3.0 to $4.5 \mathrm{~h}$ after stroke [27].

The prevalence of infection in AIS is high, ranging from $5 \%$ to $65 \%$ depending on the study population and the definition of infection [28]. It was $28.5 \%$ in our study. The most common infection in AIS is pneumonia - a recent systematic review reported a prevalence of $14.3 \%$ [29]. The prevalence of pneumonia is slightly higher in our study (17.5\%).

From the pathophysiologic point of view the high risk of infection in AIS patients is thought to be related to stroke-induced immunodepression syndrome (SIDS), which is responsible for a long-lasting alteration of the lymphocyte profile and disturbances in cytokine production [30, 31]. Additionally, the key factor specifically increasing the risk of pneumonia in AIS is dysphagia, causing oropharyngeal aspiration [32]. 


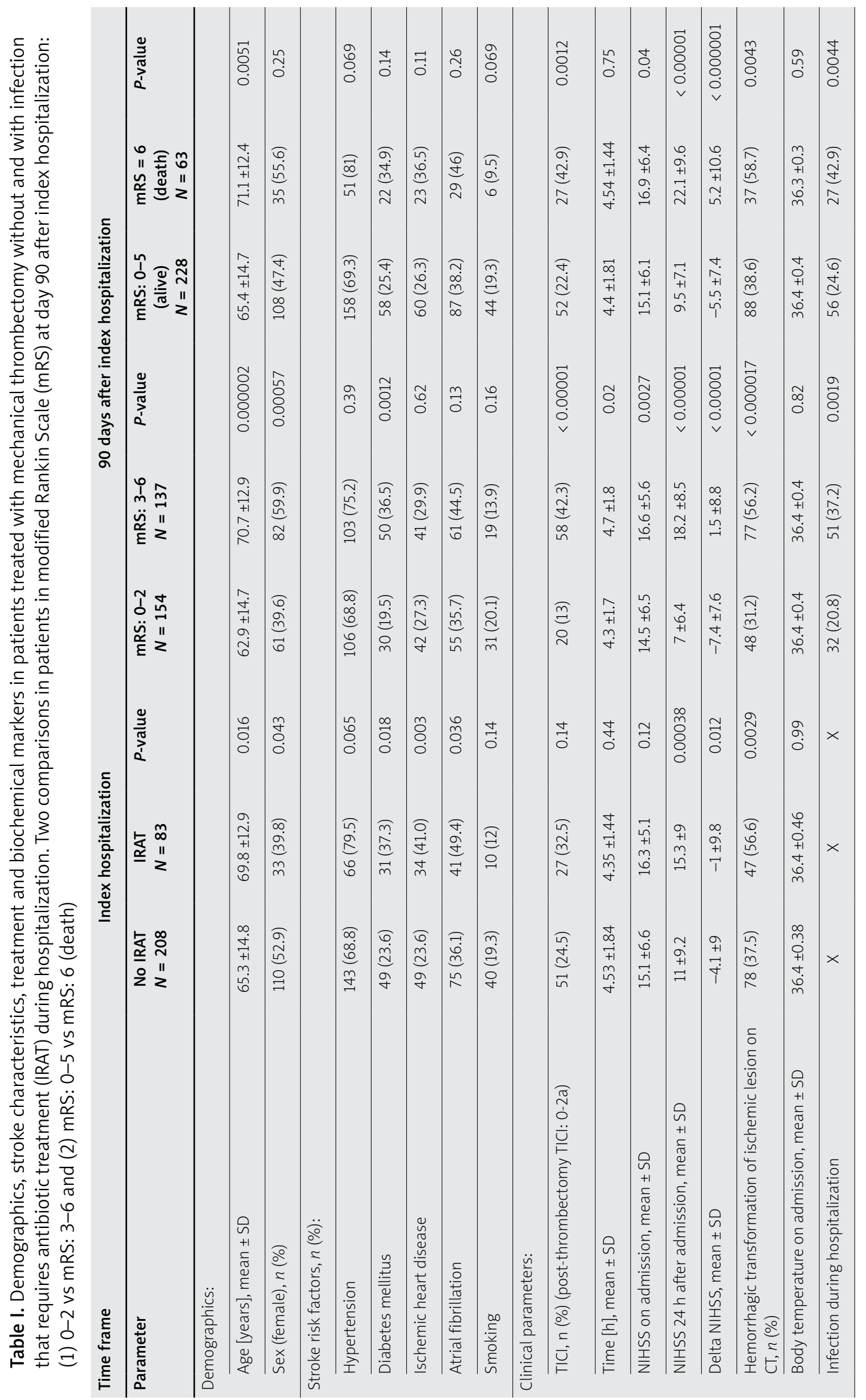




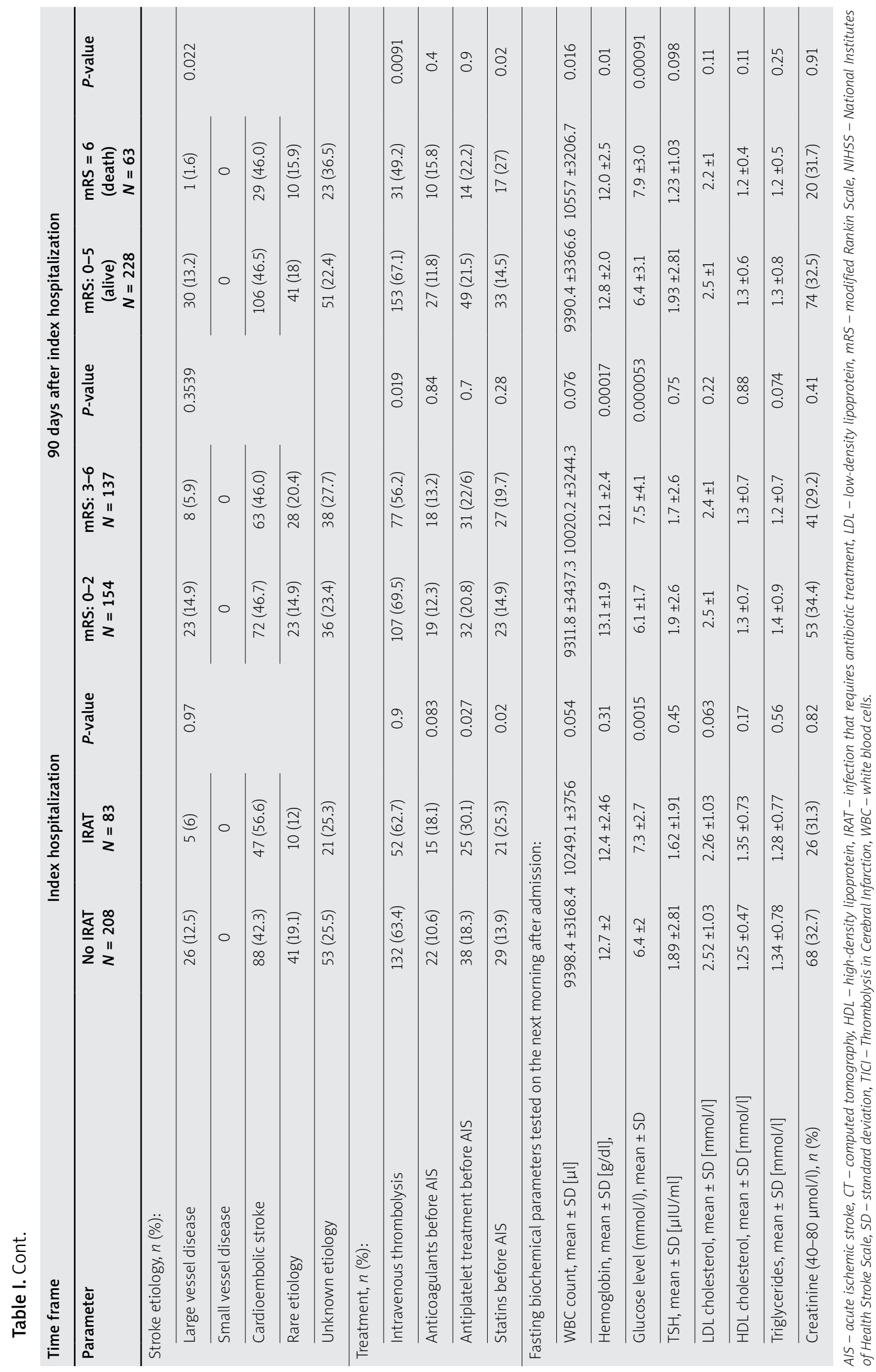


Table II. Multivariate logistic regression models showing: (1) factors affecting risk of infection that requires antibiotic treatment, (2) factors affecting favorable outcome (mRS: 0-2) at day 90 and (3) factors affecting mortality (mRS: 6) at day 90

\begin{tabular}{|c|c|c|c|}
\hline Factors affecting risk of infection that requires antibiotic treatment & OR & $95 \% \mathrm{Cl}$ & $P$-value \\
\hline \multicolumn{4}{|l|}{ Demographics: } \\
\hline Age [years] & 1.02 & $0.995-1.05$ & 0.1 \\
\hline Sex (female) & 0.39 & $0.21-0.72$ & 0.0026 \\
\hline \multicolumn{4}{|l|}{ Stroke risk factor: } \\
\hline Diabetes mellitus & 1.01 & $0.52-1.97$ & 0.97 \\
\hline Ischemic heart disease & 1.47 & $0.79-2.74$ & 0.22 \\
\hline Atrial fibrillation & 1.42 & $0.76-2.63$ & 0.27 \\
\hline \multicolumn{4}{|l|}{ Clinical parameters: } \\
\hline Delta NIHSS & 1.02 & $0.99-1.05$ & 0.18 \\
\hline Hemorrhagic transformation of ischemic lesion on CT $24 \mathrm{~h}$ after admission & 1.84 & $1.05-3.25$ & 0.034 \\
\hline \multicolumn{4}{|l|}{ Treatment: } \\
\hline Antiplatelet treatment before AIS & 1.5 & $0.68-3.31$ & 0.31 \\
\hline Statins before AIS & 1.16 & $0.5-2.73$ & 0.72 \\
\hline \multicolumn{4}{|l|}{ Biochemical parameters: } \\
\hline Fasting glucose level on the next day after admission $24 \mathrm{~h}[\mathrm{mmol} / \mathrm{ll}]$ & 1.12 & $0.98-1.27$ & 0.094 \\
\hline Factors affecting favorable outcome at day 90 (mRS: 0-2) & OR & $95 \% \mathrm{Cl}$ & $P$-value \\
\hline \multicolumn{4}{|l|}{ Demographics: } \\
\hline Age [years] & 0.95 & $0.93-0.98$ & 0.00048 \\
\hline Sex (female) & 0.46 & $0.23-0.93$ & 0.031 \\
\hline \multicolumn{4}{|l|}{ Stroke risk factors: } \\
\hline Diabetes mellitus & 0.60 & $0.28-1.29$ & 0.19 \\
\hline \multicolumn{4}{|l|}{ Clinical parameters: } \\
\hline Delta NIHSS & 0.87 & $0.82-0.91$ & 0.00000022 \\
\hline Post-thrombectomy $\mathrm{TICl}$ score: $0,1,2 \mathrm{a}$ & 0.27 & $0.12-0.58$ & 0.00086 \\
\hline Time from stroke onset to groin puncture [h] & 0.78 & $0.64-0.97$ & 0.02 \\
\hline Infection that requires antibiotic treatment & 0.72 & $0.34-1.49$ & 0.37 \\
\hline Hemorrhagic transformation of ischemic lesion on CT $24 \mathrm{~h}$ after admission & 0.47 & $0.24-0.9$ & 0.023 \\
\hline \multicolumn{4}{|l|}{ Treatment: } \\
\hline Intravenous thrombolysis & 1.33 & $0.68-2.62$ & 0.4 \\
\hline \multicolumn{4}{|l|}{ Biochemical parameters: } \\
\hline Fasting glucose level on the next day after admission $24 \mathrm{~h} \mathrm{[mmol/l]}$ & 0.93 & $0.8-1.09$ & 0.39 \\
\hline Hemoglobin [g/dl] & 1.14 & $0.97-1.34$ & 0.11 \\
\hline Factors affecting risk of death at day 90 & OR & $95 \% \mathrm{Cl}$ & $P$-value \\
\hline \multicolumn{4}{|l|}{ Demographics: } \\
\hline Age [years] & 1.04 & 1.009-1.07 & 0.010 \\
\hline Sex (female) & 0.65 & $0.30-1.42$ & 0.27 \\
\hline
\end{tabular}


Table II. Cont.

\begin{tabular}{|c|c|c|c|}
\hline Factors affecting risk of death at day 90 & OR & $95 \% \mathrm{Cl}$ & $P$-value \\
\hline \multicolumn{4}{|l|}{ Clinical parameters: } \\
\hline Post-thrombectomy TICI score: $0,1,2 a$ & 1.18 & $0.30-1.42$ & 0.68 \\
\hline Delta NIHSS & 1.17 & $1.10-1.24$ & 0.00000019 \\
\hline Hemorrhagic transformation on CT $24 \mathrm{~h}$ after admission & 2.00 & $0.96-4.20$ & 0.062 \\
\hline Infection that requires antibiotic treatment & 1.21 & $0.56-2.63$ & 0.63 \\
\hline \multicolumn{4}{|l|}{ Stroke etiology: } \\
\hline Unknown etiology of stroke & 3.55 & $1.55-8.15$ & 0.0027 \\
\hline \multicolumn{4}{|l|}{ Treatment: } \\
\hline Intravenous thrombolysis & 0.41 & $0.19-0.88$ & 0.0021 \\
\hline \multicolumn{4}{|l|}{ Biochemical parameters: } \\
\hline WBC count $[\mu l]$ & 1.10 & $0.99-1.23$ & 0.065 \\
\hline Fasting glucose level on the next day after admission $24 \mathrm{~h}[\mathrm{mmol} / \mathrm{l}]$ & 1.05 & $0.96-1.15$ & 0.31 \\
\hline
\end{tabular}

AIS - acute ischemic stroke, CI-confidence interval, CT - computed tomography, mRS - modified Rankin Scale, NIHSS - National Institutes of Health Stroke Scale, $O R$ - odds ratio, $\mathrm{TICl}$ - Thrombolysis in Cerebral Infarction, WBC - white blood cells.

The most commonly mentioned predictors of infection in AIS patients, irrespectively of their acute treatment status, are older age, sex for different type of infection, stroke severity, bedridden state, dysphagia or reduced bulbar reflexes, chronic obstructive pulmonary disease, diabetes mellitus, or specific invasive maneuvers (feeding tube placement, urinary catherization, etc.) $[6,7,24]$. The profile of factors affecting the risk of pneumonia in an unselected group of AIS patients or patients treated solely with IVT is similar to those mentioned above [8-11, 22, 23].

Many previous studies in AIS patients regardless of their acute treatment status showed that infection leads to worse outcomes. The occurrence of any post-stroke infection is associated with the odds ratio for a poor functional outcome that varied from 0.9 to 4.4 ; the odds ratio for mortality varies from 1.5 to 6.0. Regarding pneumonia, the evidence for functional outcomes is limited, and the odds ratio varies from 1.7 to 52 . For mortality, the odds ratio varies from 2.1 to 3.0 [28].

In the present study univariate analysis confirmed that infection was less frequent in patients with better prognosis and significantly more common in those who died. Interestingly, infection did not enter the logistic regression models for either prognostic outcome (favorable outcome or death at day 90). In our study, however, we confirmed the prognostic significance of several factors previously shown to be related to long-term prognosis in patients treated with MT, such as age [33], sex [34], time from stroke onset to groin puncture [35], recanalization after MT [33, 36], and hemorrhagic transformation on CT after the procedure [33]. We also observed for the first time that lower delta NIHSS - which in our study reflects the neurological improvement after the procedure - was an independent factor affecting favorable outcome at day 90. A higher delta NIHSS reflects clinical deterioration after the procedure in our study, and this score was an independent risk factor of death at day 90. Our findings showing the correlation of AIS etiology and the lack of IVT treatment before MT with 90 day mortality needs confirmation in different populations.

Our study has some limitations. First, this was a retrospective and single-center study including only patients who could give signed consent. This study was based on data acquired in a single hospital, and thus the results may be not comparable with patients collected in a multicenter prospective study. Further prospective studies are needed to validate our findings. Unfortunately, we did not collect $C$ reactive protein levels from the first 67 cases included in our study; thus, this parameter was not included in the present analysis. We are aware that this is an important factor affecting stroke outcome [37]. Finally, we did not systematically collect information about dysphagia or intubation during the procedure; both are important factors related to the risk of pneumonia.

\section{Conclusions}

In the present study infections are a common finding in patients with AIS treated with MT, and in contrast to studies on unselected AIS patients or patients treated with IVT, the presence of IRAT did not affect the studied long-term outcome measures.

\section{Acknowledgments}

The study was funded by the grant from the Jagiellonian University N41/DBS/000464. 


\section{Conflict of interest}

The authors declare no conflict of interest.

\section{References}

1. Feigin VL, Forouzanfar MH, Krishnamurth R, et al. Global and the regional burden of stroke during 1990-2010: finding from the Global Burden of Disease Study 2010. Lancet 2014; 383: 245-55.

2. Powers WJ, Rabinstein AA, Ackerson T, et al. Guidelines for the early management of patients with acute ischemic stroke: 2019 Update to the 2018 guidelines for the early management of acute ischemic stroke: a guideline for healthcare professionals from the American Heart Association/American Stroke Association. Stroke 2019; 50: e344-418.

3. Mechanical clot retrieval for treating acute ischemic stroke. National Institute for health and Care excellence (NICE). Available: https: www.nice.org.uk/guidance/ipg548

4. Nowak K, Derbisz J, Jagiełła J, et al. Time from stroke onset to groin puncture affects the rate of recanalization after mechanical thrombectomy: a real life single center experience. Neurol Neurochir Pol 2020; 54: 156-60.

5. Kumar S, Selim MH, Caplan LR. Medical complications after stroke. Lancet Neurol 2010; 9: 105-18.

6. Westendorp WF, Nederkoorn PJ, Vermeij JD, et al. Post-stroke infection. A systematic review and meta-analysis. BMC Neurol 2011; 11: 110-7.

7. Ionita CC, Gibbons KJ. Acute ischemic stroke and infections. J Stroke Cerebrovasc Dis 2011; 20: 1-9.

8. Gong S, Zhou Z, Zhou M, et al. Validation and risk scoring models for predicting stroke associated pneumonia in patients with ischemic stroke. Stroke Vasc Neurol 2016; 1: 122-6.

9. Finlayson O, Kapral MK, Hall R, et al. Stroke associated pneumonia (SAP): risk factors for its development and impact on stroke outcome. Cerebrovasc Dis 2010; 29: 190.

10. Finlayson O, Kapral M, Hall R, et al. Risk factors, inpatients care, and outcomes of pneumonia after ischemic stroke. Neurology 2011; 77: 1338-45.

11. Martino R, Foley N, Bhodal S, et al. Dysphagia after stroke: incidence, diagnosis and pulmonary complications. Stroke 2005; 36: 2756-63.

12. Ingeman A, Andersen G, Hundborg HH. In-hospital medical complications; lengths of stay, and mortality among stroke unit patients. Stroke 2011; 42: 3114-218.

13. Saxena SK, Ng TP, Yong D. Total direct cost, length of hospital stay, institutional discharges, and their determinants from rehabilitation setting in stroke patients. Acta Neurol Scan 2006; 114: 307-14.

14. Hong KS, Kang DW, Koo JS, et al. Impact of neurological and medical complications on 3-month outcomes in acute ischemic stroke. Eur J Neurol 2008; 15: 1324-31.

15. Johnston KC, Li JY, Lyden PD, et al. Medical and neurological complications of ischemic stroke: experience from RANTTAS trial. RANTTAS Investigators. Stroke 1998; 29: 447-53.

16. World Medical Association. Ethical Principles for Medical Research Involving Human Subjects JAMA 2013; 310: 2191-4.

17. WHO (World Health Organization) Cerebrovascular Disorders: a clinical a research classification. World Health Organization, Offset Publication, Geneva 1978, 43.

18. Zaidat, OO, Lazzaro MA, Liebeskind DS, et al. Revascularization grading in endovascular acute ischemic stroke therapy. Neurology 2012; 79: 110-6.
19. Brott T, Adams HP Jr, Olinger CP, et al. Measurements of acute cerebral infarction: a clinical examination scale. Stroke 1989; 20 : 864-70.

20. Adams HP Jr, Bendixen BH, LJ, Biller J, et al. 3rd Classification of subtype of acute ischemic stroke. Definitions for use in a multicenter clinical trial. TOAST. Trial of Org 10172 in Acute Stroke Treatment. Stroke 1993; 24: 35-41.

21. Van Swieten JC, Koudstaal PJ, Visser MC, et al. Interobserver agreement for the assessment of handicap in stroke patients. Stroke 1988; 19: 604-7.

22. Bruening T, Al Khaled M. Stroke-associated pneumonia in thrombolysed patients: incidence and outcome. J Stroke Cerebrovasc Dis 2015; 24: 1724-9.

23. Sheitz JF, Endres M, Heuschmann PU, et al. Reduced risk of poststroke pneumonia in thrombolyzed stroke patients with continued statin treatment. Int J Stroke 2015; 10: 61-6.

24. Rocco A, Fam G, Sykora M, et al. Poststroke infections are an independent risk factor for functional outcome after three months in thrombolysed stroke patients. Int J Stroke 2013; 8: 639-44.

25. Kalria L, Irshad S, Hodsoll J, et al. Prophylactic antibiotics in patients with dysphagia (STROKE-INF): a prospective, cluster-randomized open-label, mask endpoint, controlled clinical trial. Lancet 2015; 386: 1835-44.

26. Westendorp WP, Vermeij JD, Zock E, et al. The preventive antibiotics in stroke study (PASS): a pragmatic randomized open-label masked endpoint clinical trial. Lancet 2015; 385: 1519-26.

27. Emberson J, Lees KR, Lyden P, et al. Effect of treatment delay, age and stroke severity on the effects of intravenous thrombolysis with alteplase for acute ischemic stroke: a meta-analysis of individual patients data from randomized trials. Lancet 2014; 384: 1929-35.

28. Vermeij JD, Westendorp WF, et al. Post-stroke infections and preventive antibiotics in stroke: update and clinical evidence. Int J Stroke 2018; 13: 913-20.

29. Kishore AK, Vail A, Chamorro A, et al. How is pneumonia diagnosed in clinical stroke research? A systematic review and meta-analysis. Stroke 2015; 46: 1202-9.

30. Dirnagl U, Klehmet J, Braun JS, et al. Stroke-induced immunodepression: experimental evidence and clinical relevance. Stroke 2007; 38: 770-3.

31. Chamorro A, Urra X, Planas AM. Infection after acute ischemic stroke: manifestation of brain-induced immunodepression. Stroke 2007; 38: 1097-103.

32. Hannawi Y, Hannawi B, Rao CP, et al. Stroke-associated pneumonia: major advances and obstacles. Cerebrovasc Dis 2013; 35: 430-43.

33. Yoon W, Kim SK, Park MS, et al. Predictive factors for good outcome and mortality after stent-retriever thrombectomy in patients with acute anterior circulation stroke. J Stroke 2017; 19: 97-103.

34. Madsen TE, DeCroce-Mavson E, Hemendinger M, et al. Sex differences in 90-day outcomes after mechanical thrombectomy for acute ischemic stroke. J Neurointerv Surg 2019; 11: 221-5.

35. Raoult H, Eugene F, Ferre JC, et al. Prognostic factors for outcomes after mechanical thrombectomy with solitary stent. J Neuroradiol 2013; 40: 25-9.

36. Slater LA, Coutinho JM, Gralla J, et al. TICl and age: what's the score? AJNR Am J Neuroradiol 2016; 37: 838-43.

37. Kocatürk $M$, Kocatürk Ö. Assessment of relationship between C-reactive protein to albumin ratio and 90-day mortality in patients with acute ischaemic stroke. Neurol Neurochir Pol 2019; 53: 205-11. 